\title{
Do Rotational Shifts Affect Micturition Patterns in Real Practice? A Pilot Study in Healthy, Young Female Nurses
}

\author{
Kwang Taek Kim ${ }^{1}$, Chang Hee Kim ${ }^{1}$, Boeun Kwon², Deok Hyun Han³, Sang-Jin Yoon ${ }^{1}$, Sehyung Cho ${ }^{4}$, Kyung Jin Chung ${ }^{1}$ \\ ${ }^{1}$ Department of Urology, Gachon University Gil Medical Center, Gachon University School of Medicine, Incheon, Korea \\ ${ }^{2}$ Department of Adult Health Nursing, Seoul Women's College of Nursing, Seoul, Korea \\ ${ }^{3}$ Department of Urology, Samsung Medical Center, Sungkyunkwan University School of Medicine, Seoul, Korea \\ ${ }^{4}$ Department of Physiology, Kyung Hee University School of Medicine, Seoul, Korea
}

Purpose: Healthy, young individuals are known to exhibit circadian variation in urinary functions. However, the effects of chronic circadian disturbance on voiding functions are largely unknown. The present work compared the effects of rotational shifts on the micturition patterns of female nurses to that in female nurses with routine daytime shifts.

Methods: A total of 19 nurses without lower urinary tract symptoms who worked rotational shifts for an average duration of 2 years were recruited. A voiding diary was kept for 9 consecutive days, and the overactive bladder symptom score (OABSS) questionnaire was completed three times, starting 3 days before their night duties until 3 days after completion of their night duties. For comparison, seven nurses with regular shifts completed a 3-day voiding diary and the OABSS questionnaire.

Results: Female nurses working rotational shifts had lower overall urine production and had decreased urination frequency and nocturia than female nurses working regular shifts, even when the nurses who worked rotational shifts had a regular night's sleep for at least 7 days. Upon reinitiation of night duty, overall urine production increased significantly, with no significant changes in urgency and frequency. When these nurses returned to daytime duty, the volume of urine decreased but nocturnal urine production remained high, and the incidence of nocturia also increased significantly. However, the effects on OABSS score were not significant under the study design used.

Conclusions: Long-term rotational shifts resulted in adaptive changes such as decreased urine production and frequency in healthy, young female nurses. In addition, their micturition patterns were significantly affected by abrupt changes in their work schedules. Although working in shifts did not increase urgency or frequency of urination in healthy, young female nurses working rotational shifts for an average 2 years, large-scale studies are needed to systematically analyze the influence of shift work timings on micturition in humans.

Keywords: Circadian Rhythm; Work; Rotation; Urination

- Fund Support: This study was supported by Ferring Pharmaceuticals research fund.

- Research Ethics: All investigations were performed in accordance with the ethical guidelines of the Declaration of Helsinki, and the study protocol was approved by the Institutional Review Board of Gachon University Gil Hospital (GIRBA2834-2012).

- Conflict of Interest: No potential conflict of interest relevant to this article was reported.

\section{INTRODUCTION}

Virtually, all living things on this Earth have successfully devel- oped endogenous mechanisms that harmonize a variety of behavioral, physiological, and biochemical processes according to the repetitive changes of day and night [1,2]. The emerging cir-

Corresponding author: Kyung Jin Chung (iD http://orcid.org/0000-0003-0153-7814 Department of Urology, Gachon University Gil Medical Center, Gachon University School of Medicine, 21 Namdong-daero 774beon-gil, Namdong-gu, Incheon 405-760, Korea

E-mail: kjchung@gilhospital.com / Tel: +82-32-460-3334 / Fax: +82-32-460-8414

Submitted: October 18, 2014 / Accepted after revision: December 12, 2014 
cadian endogenous oscillators are believed to help maintain most physiological functions in mammals, including humans, according to the time of the day. These oscillators are tightly locked into the active and rest phases of a given organism in order to ensure optimal performance. While various circadian rhythms driven by the endogenous oscillators aid the maintenance of daily life, their disruption or disturbance causes disharmony of physiological functions that leads to negative health consequences. Moreover, circadian rhythmicity is sustained not by passive reactions to exogenous stimuli but by endogenous mechanisms, which are well illustrated in the rhythmic changes of various human physiological functions even under constant environmental conditions. Recent studies have demonstrated that peripheral clocks, in addition to the central clock in the suprachiasmatic nucleus, exist throughout the body, even at the cellular level [2,3].

Healthy, young individuals exhibit diurnal or circadian variation in urine volume, electrolyte excretion, urine flow, frequency, and volume per void $[4,5]$. In addition, definite diurnal differences have been observed in plasma hormonal levels, kidney excretion, and nerve stimulation that are closely related to urinary functions [5]. Although many urinary functions exhibit clear circadian variations in humans, the precise underlying mechanisms are not yet clear. Intuitively, urine production and voiding need to predominate during the active phase, whereas increased urine storage within the bladder is required to promote better sleep during the rest phase. Thus, disturbances in proper voiding rhythms, typically presented in children with nocturnal enuresis or in elderly individuals with nocturia, are predicted to negatively affect human wellbeing [6]. Indeed, nocturia, abnormal waking during the night one or more times to void, is known to decrease quality of life and negatively affect morbidity and even mortality, especially in the elderly [7]. The actual functional relationship between circadian rhythm and micturition activity is very important because urine excretion is not only closely related to body homeostasis, including psychosocial health, but also directly causes discomfort in daily life.

In industrialized societies, demands for shift workers are ever increasing, and many environmental factors such as artificial illumination, intercontinental flights, and flexible work-hour patterns disturb endogenous circadian physiological rhythms [8]. Consequently, many individuals complain of chronic fatigue, insomnia, depression, and nervousness, as well as an increased prevalence of metabolic syndrome and cardiovascular diseases $[2,9,10]$. Because nocturia is also the result of abnormal circadi- an rhythm and is one of the most common voiding symptoms in developed and developing countries [11], a better understanding of its possible causes and ways of its prevention, especially in terms of chronic circadian disturbance, are necessary.

In this pilot study, we examined the voiding patterns of female nurses working rotational shifts and compared these patterns with those of female nurses working regular shifts. This study will help facilitate the recognition of circadian rhythmic changes in voiding patterns in nurses working in shifts. In addition, we believe that this pilot study will draw immediate attention to this field and might lead to comprehensive studies that investigate the effects of chronic circadian disturbance on urinary functions and possible ways to prevent harmful consequences.

\section{MATERIALS AND METHODS}

\section{Study Design}

From September 2012 to December 2012, we recruited 19 female nurses who were assigned to the general ward and had been working rotational shifts and recruited seven female nurses who were assigned to the outpatient department and had been working regular daytime shifts. All subjects had been working for Gachon University Gil Medical Center. All investigations were performed in accordance with the ethical guidelines of the Declaration of Helsinki, and the study protocol was approved by the Institutional Review Board of Gil Hospital (GIRBA28342012). Healthy female nurses ranging in ages from 20 to 50 years, with no self-reported lower urinary tract symptoms, were included. The demographic data of the two groups of nurses are summarized in Table 1. To avoid unnecessary complications, all subjects were requested to restrict their coffee and tea intakes. In order to avoid interruption of their daily work patterns, this study was designed to adjust the participants' work schedules after obtaining written informed consent. The outline of the study is il-

Table 1. Demographic characteristics of participating nurses

\begin{tabular}{lcc}
\hline Characteristic & $\begin{array}{c}\text { Regular } \\
\text { scheduled nurses }\end{array}$ & $\begin{array}{c}\text { Rotating } \\
\text { scheduled nurses }\end{array}$ \\
\hline Gender & Female & Female \\
Age (yr), mean (range) & $35(30-43)$ & $25(21-37)$ \\
Underlying disease & None & None \\
$\begin{array}{l}\text { Consumption of coffee, } \\
\text { tea, and alcohol }\end{array}$ & Restricted & Restricted \\
$\begin{array}{l}\text { Duration of assigned work (yr), } \\
\text { mean (range) }\end{array}$ & $3(1-13)$ & $2(1-9)$ \\
\hline
\end{tabular}




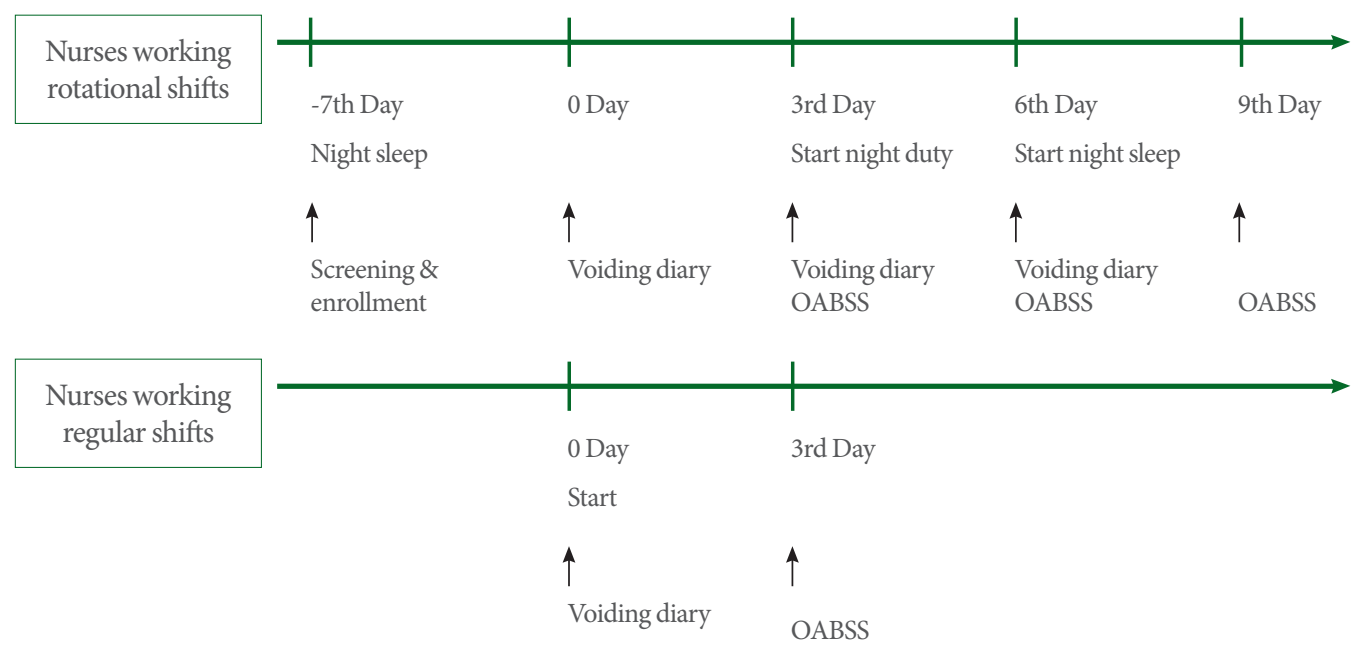

Fig. 1. Schematic outline of the study design. OABSS, overactive bladder symptom score.

lustrated in Fig. 1. For nurses working rotational shifts, the study was initiated when the participant had a minimum of 7 days of nighttime sleep, followed by 3 days of night duty and 3 days of subsequent daytime duty. Participants were asked to keep a 3-day voiding diary three times encompassing three different shift conditions, starting 3 days before a night duty. At the end of each voiding diary, nurses were asked to complete the overactive bladder symptom score (OABSS) questionnaire. Thus, a voiding diary was kept for 9 consecutive days, and the OABSS questionnaire was completed three times for each nurse working rotational shifts. For comparison, seven nurses working regular daytime shifts completed a 3-day voiding diary and the OABSS questionnaire. The total urine volume, daytime urine volume, nighttime urine volume, mean voiding volume per void, subjective urgency rating, urination frequency, functional bladder capacity, and occurrences of nocturia were evaluated from the voiding diary (Table 1, Fig. 1).

\section{Statistical Analysis}

Nurses were divided into two groups: nurses working regular shifts and nurses working rotational shifts. Values are given as means ( \pm standard deviation), and categorical data are presented as numbers and percentages (\%) where appropriate. Owing to the skewed distribution of variables, a nonparametric test (Wilcoxon rank-sum test) was used for comparison of mean values at baseline and at the follow-up assessment. A nonparametric repeated measure analysis of variance (Friedman test) was used to evaluate the statistical difference on the effect of work schedule among nurses working rotational shifts. The Wilcoxon signed- rank test was used to evaluate statistical differences between the groups.

\section{RESULTS}

Demographic data of each group of nurses are presented in Table 1 . The mean age was 25 years ( $21-37$ years) and 35 years (30-43 years), respectively, in the two groups.

First, we compared the voiding patterns between nurses working regular shifts and nurses who had been working rotational shifts for at least 1 year and had regular night's sleep for at least 7 days. As shown in Table 2, the total daytime and nighttime urine volumes were significantly lower in nurses who had previously worked rotational shifts for varying durations, indicating that urine production decreased in nurses working rotational shifts. Accordingly, mean urine volume per void and voiding frequency were also significantly lower than those in nurses working regular shifts. However, no statistically significant differences were observed in subjective urgency rating, functional bladder capacity, or OABSS score. These data suggest that long-term exposure to rotational shifts may decrease urine production even in healthy female nurses working rotational shifts. As shown in Fig. 2, daily total urine volume was significantly lower in nurses working rotational shifts, even when they had regular night's sleep for at least 7 days, as compared to nurse on regular shifts (Table 2, Fig. 2).

Next, we examined changes in micturition-related parameters before, during, and after night duty in the rotational shift-working nurses (Table 3). The total and daytime amount of urine vol- 
Table 2. Comparison of voiding patterns during daytime schedule between nurses working a regular schedule and nurses who had been previously exposed to the rotational shift-work schedule for at least one year

\begin{tabular}{lccc}
\hline Variable & Regular scheduled nurses & Rotating scheduled nurses & P-value \\
\hline Total amount of urine volume $(\mathrm{mL})$ & $1,276.90 \pm 211.21$ & $961.20 \pm 263.98$ & $0.010^{*}$ \\
Daytime amount of urine volume $(\mathrm{mL})$ & $1,226.43 \pm 265.32$ & $927.31 \pm 263.54$ & $0.028^{*}$ \\
Nighttime amount of urine volume $(\mathrm{mL})$ & $50.48 \pm 100.65$ & $33.89 \pm 56.49$ & $0.049^{*}$ \\
Mean voiding volume (mL/voiding) & $252.73 \pm 69.99$ & $222.20 \pm 79.72$ & $0.006^{*}$ \\
Urgency rate & $2.23 \pm 0.55$ & $2.06 \pm 0.65$ & 0.579 \\
Frequency (times) & $5.24 \pm 0.96$ & $4.59 \pm 1.32$ & $0.020^{*}$ \\
Nocturia (times) & $0.19 \pm 0.29$ & $0.14 \pm 0.26$ & $0.037^{*}$ \\
Functional bladder capacity $(\mathrm{mL})$ & $381.43 \pm 58.15$ & $388.06 \pm 129.83$ & 0.067 \\
OABSS & $1.43 \pm 1.13$ & $1.28 \pm 1.32$ & 0.527 \\
\hline
\end{tabular}

Values are presented as mean \pm standard deviation.

OABSS, overactive bladder symptom score.

${ }^{\text {a) }}$ Subjective symptom score $(1-5) .{ }^{*} \mathrm{P}<0.05$, statistically significant differences between the groups.

\begin{tabular}{|c|c|c|}
\hline $\begin{array}{c}\text { Initial daytime shift } \\
\text { (days 1-3) }\end{array}$ & $\begin{array}{c}\text { Rotational nighttime shift } \\
\text { (days 4-6) }\end{array}$ & $\begin{array}{c}\text { Rotational daytime shift } \\
\text { (days 7-9) }\end{array}$ \\
\hline
\end{tabular}

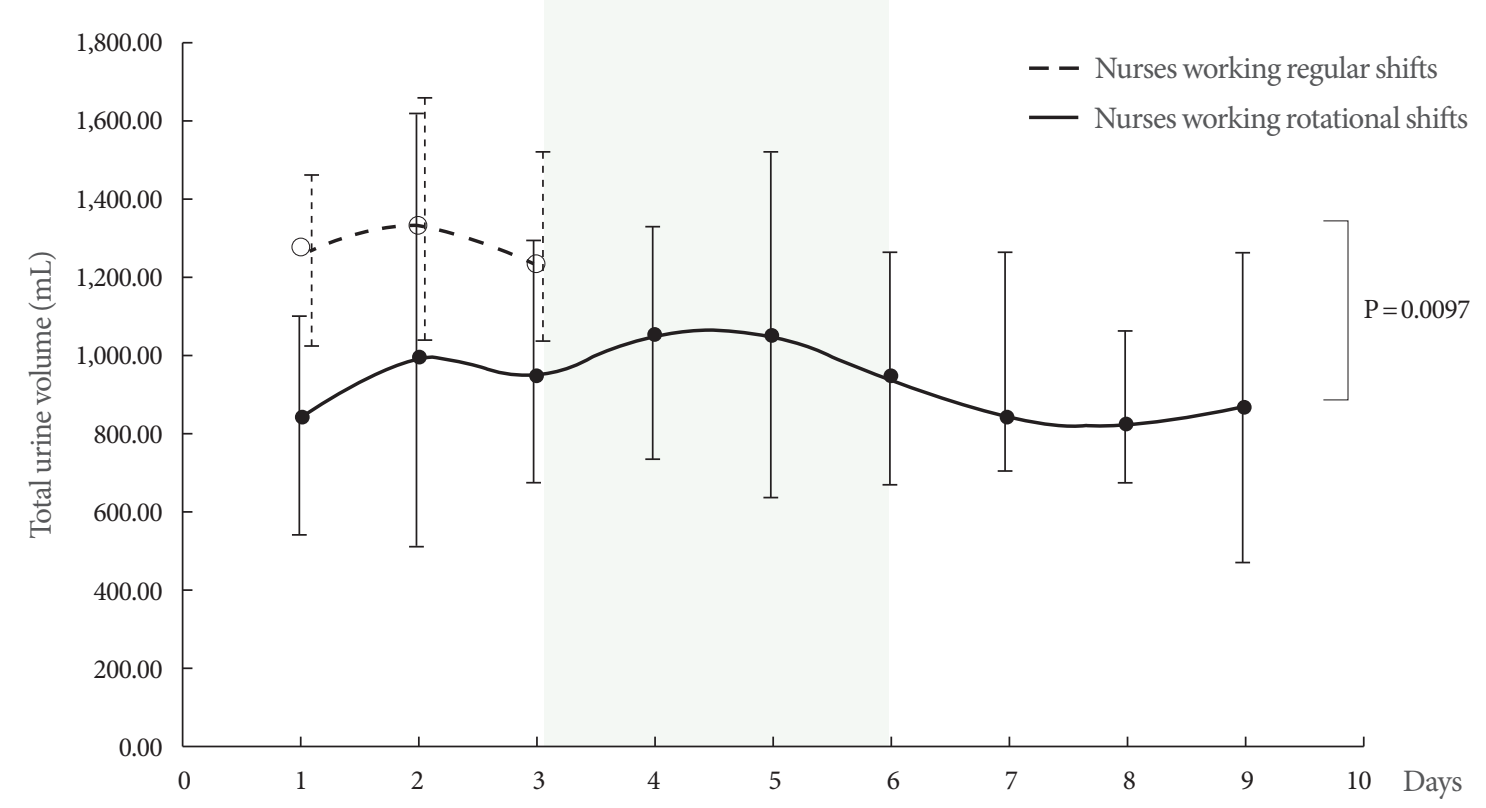

Fig. 2. Mean ( $\pm 95 \%$ confidence intervals) of total amount of urine volume in nurses working a regular schedule (open circle) and nurses working a rotating schedule (closed circle). $\mathrm{P}=0.010$ between two groups during the first three days.

ume was increased upon reinitiation of night duty and returned to lower levels after cessation of night duty. Accordingly, mean urine volume per void was increased upon initiation of night duty, while functional bladder capacity seemed to be slightly decreased. Interestingly, nighttime amount of urine volume and nocturia incidence were significantly increased upon returning to daytime duty, suggesting that nocturnal production of urine persists at least for 3 days even when they were engaged in daytime duty. However, we found no statistical difference in subjective urgency rating, urination frequency, or OABSS score for the entire recording period. These data suggest that abrupt changes of work schedule significantly affect urine volume-related parameters as well as nocturnal urination. Yet, no effects on actual discomfort-related parameters such as frequency or urgency 
Table 3. Comparison of voiding patterns before, during, and after night duty in rotational shift-working nurses

\begin{tabular}{|c|c|c|c|c|}
\hline Variable & Initial daytime shift (A) & Rotating nighttime shift (B) & Rotating daytime shift (C) & P-value \\
\hline Total amount of urine volume $(\mathrm{mL})$ & $961.20 \pm 263.98$ & $1,014.90 \pm 313.12$ & $905.74 \pm 191.42$ & $<0.05^{*}$,b) \\
\hline Daytime amount of urine volume $(\mathrm{mL})$ & $927.31 \pm 263.53$ & $1,014.90 \pm 313.12$ & $842.04 \pm 222.00$ & $<0.05^{*, \mathrm{~b})}$ \\
\hline Nighttime amount of urine volume $(\mathrm{mL})$ & $33.89 \pm 56.49$ & - & $63.70 \pm 81.86$ & $<0.05^{*, c)}$ \\
\hline Mean voiding volume (mL/voiding) & $222.20 \pm 79.72$ & $236.23 \pm 77.42$ & $209.04 \pm 66.68$ & $\left.<0.05^{*}, \mathrm{~b}\right)$ \\
\hline Urgency rate $e^{a}$ & $2.06 \pm 0.64$ & $2.16 \pm 0.64$ & $2.12 \pm 0.63$ & $>0.05^{\mathrm{b})}$ \\
\hline Frequency (times) & $4.59 \pm 1.32$ & $4.54 \pm 1.28$ & $4.61 \pm 0.94$ & $>0.05^{\mathrm{b})}$ \\
\hline Nocturia (times) & $0.14 \pm 0.26$ & - & $0.39 \pm 0.56$ & $<0.05^{*, c)}$ \\
\hline Functional bladder capacity $(\mathrm{mL})$ & $388.06 \pm 129.83$ & $344.45 \pm 97.27$ & $369.44 \pm 114.15$ & $<0.05^{*, \mathrm{~b})}$ \\
\hline OABSS & $1.28 \pm 1.32$ & $1.83 \pm 1.69$ & $1.56 \pm 1.42$ & $>0.05^{\mathrm{b})}$ \\
\hline
\end{tabular}

Values are presented as mean \pm standard deviation. The value were evaluated with the post hoc test.

OABSS, overactive bladder symptom score.

a) Subjective symptom score (1-5). ${ }^{\text {b) }} \mathrm{A}$ vs. B vs. C. ${ }^{\mathrm{c}} \mathrm{A}$ vs. C. ${ }^{\star} \mathrm{P}<0.05$, statistically significant differences between the groups.

were observed in healthy rotational shift-working female nurses (Table 3).

\section{DISCUSSION}

Micturition is one of the most important physiological functions, as it excretes waste and regulates body homeostasis. Indeed, abnormal micturition patterns directly affect comfort in daily lives. In this pilot study, we observed that overall urine production decreased significantly in healthy nurses working rotational shifts compared to that in nurses who work regular shifts, even when nurses who worked night shifts had regular night's sleep for at least 7 days. Accordingly, urination frequency and nocturnal urination also decreased without any significant changes in subjective urgency rating, functional bladder capacity, or OABSS score. Upon reinitiating their night duty, overall urine production increased significantly and functional bladder capacity decreased. When these returned to daytime duty, their urine production was markedly lowered, but their nighttime urine production remained high and nocturnal urination was significantly elevated. These results suggest that rotational shifts cause adaptive changes in micturition, and that micturition patterns are affected by shift work. However, the precise mechanisms underlying these adaptive changes remain to be elucidated.

In fact, multiple mechanisms serve to maintain micturition function. In the kidney, mechanisms are adjusted according to the degree of metabolism. For example, metabolism decreases following decreases in urine production during sleep and increases following increases in urine output, which results in an increase in glomerular filtration rate during the daytime [12]. The major role of the urinary bladder is to act as a reservoir during the night/rest phase and to act as an excretory organ during the day/active phase $[13,14]$. Accordingly, complex interactions between the bladder and kidney may affect the frequency of micturition and the volume of urine output during the day and night. One study reported that sleep deprivation leads to diuresis and natriuresis [15]. However, the causative factors that lead to changes in voiding behavior and the extent to which these factors affect daily micturition have not yet been clarified. In some studies, micturition count was increased during night shifts $[16,17]$. However, to the best of our knowledge, no study has reported on the actual voiding patterns of individuals with rotating shifts. Proper understanding of the relationship between rotational shifts and voiding rhythm is very important because micturition is not only essential for maintaining homeostasis but also directly affects personal daily comfort in daily life. Furthermore, discomfort caused by nocturia or frequency also exerts a negative influence on the circadian rhythm and vice versa. Nocturia is one of the most frequent and bothersome symptoms of disturbed circadian rhythm in men and women. It influences sleep architecture at night [18] that leads to increased risk of falling, fracture, daytime somnolence, and even mortality, particularly in the elderly [19].

It is not easy to conduct a clinical study in human subjects to assess changes in micturition patterns according to rotational shifts, primarily owing to the difficulty of recruiting suitable subjects and ensuring appropriate study circumstances. In addition, study subjects must quantify the urine volume and fill out the voiding diary at each void, which is quite inconvenient and 
can be easily forgotten. Furthermore, micturition is affected by multiple factors such as psychological, hormonal, neuronal, renal, and bladder-related factors such as storage and excretory functions. Although some studies on the relationship between circadian rhythm and renal function have been reported [12], there are very few studies on the relationship between circadian disturbance and micturition. One report addressed nighttime frequency after circadian disturbance [16], but the results were very fragmentary, as they were solely based upon a questionnaire. In a recent study, normal adults showed a greater voiding volume per micturition at night and decreased maximal flow rate [20,21]. Differences between daytime and nighttime bladder capacities and frequencies have also been reported in rats, further suggesting that bladder function might be a direct target of circadian regulation [22]. On the other hand, fixed bladder capacity and voiding frequency have also been reported, irrespective of circadian disturbance [22].

Our study reveals new insights on the effect of rotational shifts on micturition. Although urine volume-related parameters increased with reinitiation of night duty, its actual effect on discomfort was found marginal in healthy, young female nurses. This suggests that the urinary bladder may adjust urgency and frequency according to rotational shifts. In addition, while abrupt changes in work schedule alter micturition patterns, long-term exposure to an irregular work schedule, i.e., rotational shifts, may conversely result in decreased urine production in humans. The findings obtained in this pilot study are novel because most studies on nurses' night shifts were confined to correlations between shift work and risk of cancer, which are related to changes in hormones such as melatonin and catecholamine [23-26]. To elucidate the relationship between shift work timings and micturitional changes, additional large-scale studies are needed to fully encompass physiological and psychosocial variables. Another important point is that decrease in urine production was observed in nurses working rotational shifts, which may decrease renal function and/or increase the risk of urinary stones.

Other factors need to be considered when analyzing altered micturition patterns in female subjects. Examples are their menstruation cycles, daily consumption of food and fluid intake, and daily intake of caffeine-containing beverages such as coffee and tea. Shift work interval and the actual duration of assignment to rotational shifts might also affect the outcome. However, owing to the small sample size in this pilot study, we could not assess these variables. Thus, effects of these putative factors need to be addressed in subsequent large-scale studies.
Other limitations to this pilot work are also worth mentioning. First, this study was designed to accommodate nurses' daily schedules to minimize interruptions in participants' daily jobs. Consequently, the 3-day rotational interval might be too short to observe full adaptive changes related to shift work. Second, the majority of individuals engaged in rotational shifts, such as nurses, police officers, or fire fighters, often perform emergent duties or are very busy, so each urination can easily be affected by their daily circumstances or workloads. Therefore, alterations in micturition patterns observed in this pilot study might be also affected by these variables. Third, atypical fragmented sleep patterns during daytime sleep make it difficult to define or quantify voiding parameters such as nocturia. Thus, nocturia during daytime sleep was not evaluated in this study. Fourth, the effect of chronic circadian disturbance on the incidence of nocturia was the primary interest of our study; however, we could not identify remarkable changes. This can be attributed to the fact that shift work jobs are usually assigned to healthy, young nurses who have relatively rare episodes of nocturia. Nevertheless, we found their nighttime urine production remained high and incidence of nocturia significantly increased upon returning to daytime duty, although the degree was quite small.

Further, nurses working rotational shifts often complain about atypical fragmented sleep patterns in their daytime sleep schedule. For example, nurses who completed overnight duties sleep in the mornings for several hours and have short daily activities, and additive short sleep before the next night duty. This fragmented sleep pattern might negatively influence the quality of sleep and the general state of health. Therefore, it is important to assess health-related long-term effects of altered micturition and sleep pattern in rotational shift workers, such as nurses, fire fighters, and police officers. Further studies are needed to elucidate precise mechanisms that alter urine output and urination patterns in rotational shift workers.

In conclusion, long-term exposure to a rotational shifts resulted in adaptive changes such as decreased urine production and urination frequency in healthy, young female nurses. However, micturition patterns were significantly affected by changes in schedule of nurses working in shifts. However, shift work did not cause urgency or frequency in healthy, young female nurses who worked rotational shifts for an average 2 years. Further large-scale studies are needed to systematically analyze the negative influences of chronic circadian disturbance on micturition functions and to find possible ways to prevent these influences. 


\section{ACKNOWLEDGEMENTS}

The authors thank to Ju Hee Hwang (Research nurse of Department of Urology, Gachon University Gil Medical Center, Incheon, Republic of Korea) for interviewing and instructing the study participants.

\section{REFERENCES}

1. Levi F, Schibler U. Circadian rhythms: mechanisms and therapeutic implications. Annu Rev Pharmacol Toxicol 2007;47:593-628.

2. Takahashi JS, Hong HK, Ko CH, McDearmon EL. The genetics of mammalian circadian order and disorder: implications for physiology and disease. Nat Rev Genet 2008;9:764-75.

3. Dibner C, Schibler U, Albrecht U. The mammalian circadian timing system: organization and coordination of central and peripheral clocks. Annu Rev Physiol 2010;72:517-49.

4. Cho S, Han DH, Kim DH. Circadian rhythms in voiding function and dysfunction. Int Neurourol J 2011;15:2-3.

5. Noh JY, Han DH, Yoon JA, Kim MH, Kim SE, Ko IG, et al. Circadian rhythms in urinary functions: possible roles of circadian clocks? Int Neurourol J 2011:15:64-73.

6. Neveus T. Diagnosis and management of nocturnal enuresis. Curr Opin Pediatr 2009;21:199-202.

7. Kujubu DA, Aboseif SR. An overview of nocturia and the syndrome of nocturnal polyuria in the elderly. Nat Clin Pract Nephrol 2008;4:426-35.

8. Costa G. The 24-hour society between myth and reality. J Hum Ergol (Tokyo) 2001;30:15-20.

9. Takeda N, Maemura K. Circadian clock and cardiovascular disease. J Cardiol 2011;57:249-56.

10. Kohyama J. Neurochemical and neuropharmacological aspects of circadian disruptions: an introduction to asynchronization. Curr Neuropharmacol 2011;9:330-41.

11. Irwin DE, Milsom I, Hunskaar S, Reilly K, Kopp Z, Herschorn S, et al. Population-based survey of urinary incontinence, overactive bladder, and other lower urinary tract symptoms in five countries: results of the EPIC study. Eur Urol 2006;50:1306-14.

12. Koopman MG, Krediet RT, Arisz L. Circadian rhythms and the kidney. A review. Neth J Med 1985;28:416-23.

13. Van Hoeck K, Bael A, Lax H, Hirche H, van Gool JD. Circadian variation of voided volume in normal school-age children. Eur J Pediatr 2007;166:579-84.

14. Ku JH, Lim DJ, Byun SS, Paick JS, Oh SJ. Nocturia in patients with lower urinary tract symptoms: association with diurnal voiding patterns. BJU Int 2004;93:1005-8.

15. Kamperis K, Hagstroem S, Radvanska E, Rittig S, Djurhuus JC. Excess diuresis and natriuresis during acute sleep deprivation in healthy adults. Am J Physiol Renal Physiol 2010;299:F404-11.

16. Sen RN, Kar MR. Circadian rhythms in some groups of Indians working in shifts. J Hum Ergol (Tokyo) 1978;7:65-79.

17. Bendtsen AL, Andersen JR, Andersen JT. Infrequent voiders syndrome (nurses bladder). Prevalence among nurses and assistant nurses in a surgical ward. Scand J Urol Nephrol 1991;25:201-4.

18. Kim SO, Choi HS, Kim YJ, Kim HS, Hwang IS, Hwang EC, et al. Impact of nocturia on health-related quality of life and medical outcomes study sleep score in men. Int Neurourol J 2011;15:82-6.

19. Asplund R. Mortality in the elderly in relation to nocturnal micturition. BJU Int 1999;84:297-301.

20. Poulsen EU, Kirkeby HJ. Home-monitoring of uroflow in normal male adolescents. Relation between flow-curve, voided volume and time of day. Scand J Urol Nephrol Suppl 1988;114:58-62.

21. Satoh W, Nakada T. Characteristics of circadian change in urinary frequency, bladder capacity and residual urine volume in elderly men with lower urinary tract symptoms. Nurs Health Sci 1999;1: 125-9.

22. Herrera GM, Meredith AL. Diurnal variation in urodynamics of rat. PLoS One 2010;5:e12298.

23. Davis S, Mirick DK. Circadian disruption, shift work and the risk of cancer: a summary of the evidence and studies in Seattle. Cancer Causes Control 2006;17:539-45.

24. Schernhammer ES, Laden F, Speizer FE, Willett WC, Hunter DJ, Kawachi I, et al. Rotating night shifts and risk of breast cancer in women participating in the nurses' health study. J Natl Cancer Inst 2001;93:1563-8

25. Schernhammer ES, Laden F, Speizer FE, Willett WC, Hunter DJ, Kawachi I, et al. Night-shift work and risk of colorectal cancer in the nurses' health study. J Natl Cancer Inst 2003;95:825-8.

26. Davis S, Mirick DK, Chen C, Stanczyk FZ. Night shift work and hormone levels in women. Cancer Epidemiol Biomarkers Prev 2012; 21:609-18 